\title{
Effects of Dimethyl Sulfoxide on Lipid Membrane Electroporation
}

\author{
M. Laura Fernández, ${ }^{1,2,3}$, Ramon Reigada \\ ${ }^{1}$ Laboratorio de Sistemas Complejos, Departamento de Computación, Facultad \\ de Ciencias Exactas y Naturales, Universidad de Buenos Aires, Buenos Aires, Argentina \\ ${ }^{2}$ Consejo Nacional de Investigaciones Científicas y Técnicas, Buenos Aires, Argentina \\ ${ }^{3}$ Instituto Tecnológico de Buenos Aires, Buenos Aires, Argentina \\ ${ }^{4}$ Department de Química Física and Institut de Química Teòrica i Computacional (IQTCUB), \\ Universitat de Barcelona, Barcelona, Spain
}

"CORRESPONDING AUTHOR E-MAIL ADDRESS: reigada@ub.edu

TITLE RUNNING HEAD: DMSO effect on membrane electroporation.

ABBREVIATIONS: DMSO, dimethyl sulfoxide; MD, molecular dynamics; Chol, cholesterol 
ABSTRACT. Pores can be generated in lipid membranes by the application of an external electric field or by the addition of particular chemicals such as dimethyl sulfoxide (DMSO). Molecular dynamics (MD) has been shown to be a useful tool for unveiling many aspects of pore formation in lipid membranes in both situations. By means of MD simulations, we address the formation of electropores in cholesterol-containing lipid bilayers under the influence of DMSO. We show how a combination of physical and chemical mechanisms leads to more favorable conditions for generating membrane pores and, in particular, how the addition of DMSO to the medium significantly reduces the minimum electric field required to electroporate a lipid membrane. The strong alteration of membrane transversal properties and the energetic stabilization of the hydrophobic pore stage by DMSO provide the physicochemical mechanisms that explain this effect.

Keywords: Electroporation, Molecular Dynamics, Lipid Membrane, Dimethyl sulfoxide, Electric Field

\section{Introduction}

One of the main functions of the cell membrane is to regulate the traffic of molecules from the cytoplasm to the outer cellular medium and vice versa. Transmembrane trafficking occasionally involves the formation of water pores that traverse the lipid membrane bilayer. Reversible pores in cell membranes can be physically induced by the application of an external electric field. This 
technique is referred as electropermeabilization or electroporation ${ }^{1}$ and has been used in numerous applications in biotechnology and medicine. For example, it is a common technique for introducing genetic material into cells and for delivering drugs across cell membranes. ${ }^{2}$ The latter has resulted in a new tool to battle cancer, electrochemotherapy, which is based on the application of pulses that induce the electroformation of reversible pores in the cell membrane, thereby allowing the delivery of non-permeant drugs inside tumor cells. ${ }^{2,3}$ Membrane pores can also be chemically induced by the use of particular membrane-fluidizing compounds such us dimethyl sulfoxide (DMSO, $\left(\mathrm{CH}_{3}\right)_{2} \mathrm{SO}$ ), a small amphiphilic molecule that has been shown to enhance cell permeability through the formation of water pores. ${ }^{4,5}$

Recently, molecular dynamics (MD) simulations have been used to elucidate the molecular mechanisms leading to pore formation in simple lipid bilayers by both the application of an external electric field ${ }^{6-10}$ and the effects of DMSO ${ }^{5,10-12}$ According to the simulations, the molecular mechanism of electroporation occurs in different stages. First, the disordering of the water molecules at the membrane interface due to the applied field eventually generates a water defect/intrusion that initiates pore formation. ${ }^{13}$ Such water defects can develop into water columns spanning the bilayer (hydrophobic pores), and later in the process, the lipid headgroups reorient to cover the initial water column and form a stable hydrophilic pore. ${ }^{6-10,14}$ Although this is the most common behavior, some simulations have also revealed the existence of long-lived hydrophobic pores when using high cholesterol fractions ${ }^{15}$ or negatively charged lipids. ${ }^{16}$ Simulations performed for bilayers made of different lipid compositions have revealed the role of the membrane physical properties in the electroporation process. Mainly, the electroporation threshold (the minimum electric field required to porate the membrane) is determined by the 
membrane physical properties: more stable, compact and condensed bilayers require higher applied electric fields in order to be porated..$^{9,17,18}$

MD simulations also show that in the absence of any applied electric field, DMSO triggers a similar chain of events by promoting water defects that initiate hydrophobic water columns that later develop into stable hydrophilic membrane pores. ${ }^{5,10-12}$ Because the physical (electroporation) and chemical (DMSO-induced) methods follow a similar sequence for pore formation, the combination of both acting simultaneously is expected to lead to pore formation under more favorable conditions. Actually, the increase of electroporation efficiency by DMSO has been experimentally observed. ${ }^{19}$ We contribute to this issue by investigating at the molecular level the origin of how both chemical and physical mechanisms generate pores in lipid membranes. Through MD simulations we address the formation of electropores in lipid bilayers under the influence of DMSO. Since cholesterol ( $\mathrm{Chol})$ is fundamental for determining many properties of the cell membrane and, among others, its resistance to be electroporated, ${ }^{9}$ the understanding of the action of DMSO in electroporated living membranes requires the study of Chol-containing lipid bilayers. To do so, we have conducted a systematic investigation and characterization of this phenomenon for different membrane compositions (0, 20 and $40 \mathrm{~mol} \%$ cholesterol fraction) and DMSO contents $(0,1,2.5$ and $5 \mathrm{~mol} \%$ fraction in the solvent medium). We show that electroporation is facilitated in the presence of DMSO; the electroporation threshold ${ }^{8,9,17,18}$ notably decreases upon the addition of DMSO, and this effect is significant even at small (1 mol\%) DMSO fractions in the solvent. This result is found to be consistent regardless of the Chol contents of the membrane. The numerical simulations also reveal the physicochemical mechanism leading to this effect, which is also reported. 


\section{Methods}

\subsection{Computational Methods}

MD simulations were run for dioleoylphosphatidylcholine (DOPC) bilayer systems mixed with 0, 20 and 40 mol\% cholesterol. Each bilayer was composed of 128 DOPC molecules and 0, 32 and 86 Chol molecules distributed equally and randomly in the two leaflets. Simulations were carried out using the GROMACS 3.3.1 software package. ${ }^{20}$ For DOPC molecules we used the standard united-atom force-field parameters developed by Berger et al. ${ }^{21}$ and the adaptation performed in Ref. 22 for the double bond region. The Simple Point Charge (SPC) model ${ }^{23}$ was employed for water and the force-field description of cholesterol is adapted from Ref. 24 . The SETTLE $^{25}$ and LINCS $^{26}$ algorithms were used to preserve the bond lengths in the water and lipid molecules, respectively. A single $1.0 \mathrm{~nm}$ cutoff distance was used for the Lennard-Jones interactions. The particle-mesh Ewald method ${ }^{27}$ with a real space cutoff of $1.0 \mathrm{~nm}$ was used to handle electrostatic interactions. Periodic boundary conditions were used in all three directions, and the time step was set to 2 fs.

Simulations were carried out in the NpT ensemble at $\mathrm{p}=1$ atm and $\mathrm{T}=310 \mathrm{~K}$ both controlled by using the weak coupling method. ${ }^{28}$ The pressure coupling was applied separately in the bilayer plane $(\mathrm{x}, \mathrm{y})$ and the perpendicular direction $(\mathrm{z})$. The simulation protocol applied here has been successfully used in previous MD simulations, and the obtained values for structural membrane properties in the absence of an external electric field, such as the area per molecule, the membrane thickness, and the scattering form factors are consistent with experimental data for DOPC and DOPC/Chol bilayers. ${ }^{29}$ 


\subsection{Addition of DMSO}

Each bilayer system was initially run with $0,1,2.5$ and 5 mol\% DMSO in the solvent phase. In all cases, the number of solvent molecules was fixed at 6186, but the molar fraction of DMSO in the water/DMSO mixture was varied. The DMSO force-field description developed by Bordat et al. ${ }^{30}$ was used. DMSO molecules were added to the simulated membrane systems in three different manners: randomly in the whole simulation box, at the water/membrane interface or in the bulk aqueous phase. In all three cases, DMSO molecules were partially absorbed by the bilayer, and the equilibrium was reached when the DMSO density profiles were independent of the initial insertion place and remained constant after a few nanoseconds. All simulated bilayer systems were equilibrated at $20 \mathrm{~ns}$ after the addition of DMSO. It is important to notice here that since DMSO is partly absorbed by the lipid bilayer, the final (equilibrium) DMSO molar fraction in the solvent phase is lowered respect to the initial one. The equilibrium DMSO molar fraction has been computed at equilibrium by considering the molar DMSO/solvent ratio exclusively at the solvent phase (delimitated by the average location in the z-axis of the phosphate groups at both leaflets). As an example, after DMSO absorption, the initial 1, 2.5 and 5 mol\% DMSO ratios change to $0.37,1.07$ and $2.89 \mathrm{~mol} \%$, respectively, for the bilayers containing $20 \mathrm{~mol} \%$ Chol. For clarity, however, in the remaining of the paper the simulations with DMSO are identified by its initial DMSO/solvent molar ratio.

\subsection{Measurement of the minimum porating field}

Once equilibrated, the membranes were subjected to an electric field in the direction normal to the membrane $(\mathrm{x}, \mathrm{y})$ plane. In this paper, we use high electric fields (of the order of $10^{8} \mathrm{~V} / \mathrm{m}$ ) so we are in fact modelling the effects of electroporation protocols based on nanosecond, megavolt- 
per-meter pulses. In these conditions, pulses are too short to accumulate ions and charge the membrane capacitor, so that the simulations are performed in the absence of salts. Electroporation protocols based on low-field and longer pulses result in the accumulation of ions at the cell membrane. MD simulations that mimic this protocol assume a pre-organized membrane system with an initial charge imbalance. A detailed discussion on this issue can be found in Refs. 15 and 31.

Previous MD simulations showed that a minimum electric field is required to break the bilayer configuration and form a stable water pore, and such minimum field is mainly determined by the composition of the membrane., ${ }^{9,17,18}$ Here, the electroporation threshold $\mathrm{E}_{0}$ was computed following a similar procedure to that in Ref. 9. Each membrane simulation was run independently for $25 \mathrm{~ns}$ in triplicate, and a transverse electric field, E, was fixed in the MD simulation protocol. In the case that none of the three replicas of the system displayed pore formation after $25 \mathrm{~ns}$, the field was increased by $25 \mathrm{mV} / \mathrm{nm}$ and so on. When at least one of the three replicas was porated, the value of the applied field, $\mathrm{E}$, was taken as $\mathrm{E}_{0}$. Except in cases in which the three simulations showed pore formation, a fourth replica was run at the former lower value of E. At this point it is worth noticing that given the stochastic nature of electroporation, the interpretation of the computed values of $E_{0}$ has to be taken with care. In particular, it could be expected that lower electroporation thresholds would be obtained if simulations of larger systems and/or to longer times and/or more replicas were carried out. The simulations presented here were run for time, bilayer sizes and number of replicas allowed by the available computational resources. In any case, the values of the electroporation threshold presented here for different membrane compositions are reliable enough to be used for comparative purposes. 
For completeness we also calculated the voltage $\mathrm{V}_{0}$ generated by the electroporation field; $V_{0}=E_{0} L_{z}$, where $L_{z}$ is the averaged size of the simulation box in the ' $\mathrm{z}$ '-axis when the electric field is applied.

\subsection{Measurement of membrane properties}

To analyze the alterations of the membrane structure due to the presence of DMSO and the application of an electric field, we focused on the transverse structure of the simulated membrane systems, and in particular on the mass density, lateral pressure and electrostatic potential transverse profiles. Cross-sectional profiles were computed by averaging the property of interest in the whole system as a function of the bilayer normal direction (' $z$ '-profiles). All of the ' $z$ 'profiles presented in this paper were computed by dividing the system into 0.1-nm-thick slices along the z-axis. Each property profile was measured and averaged before addition of DMSO, after the addition of DMSO and/or after application of the electric field. If membrane poration occurred, the profiles were computed before the pore was formed.

Mass density profiles, $\rho_{\mathrm{i}}(\mathrm{z})$, for different molecules and/or lipid groups ' $\mathrm{i}$ ' provide information about the distribution of the system components along the ' $z$ '-axis. The membrane electric potential profile, $\mathrm{V}(\mathrm{z})$, was computed for each membrane system as the double integral of its charge density distribution $\rho_{\mathrm{q}}(\mathrm{z})$; namely, by twice integrating the Poisson equation:

$$
\frac{d^{2} V(z)}{d z^{2}}=-\frac{\rho_{q}(z)}{\varepsilon_{0}},
$$

where $\varepsilon_{0}$ is the vacuum permittivity. Following the procedure detailed in Ref. 32, the potential and electric field were chosen to be zero at the center of mass of the bilayer $(\mathrm{z}=0)$ as boundary 
conditions for the integration that was, in turn, performed following the outward z-direction for each one of the leaflets. The lateral pressure profile, $\mathrm{P}_{\mathrm{L}}(\mathrm{z})$, was computed as the lateral components of the pressure tensor, $\left(\mathrm{P}_{\mathrm{xx}}+\mathrm{P}_{\mathrm{yy}}\right) / 2$, at different $\mathrm{z}$ values. Technically, $\mathrm{P}_{\mathrm{L}}(\mathrm{z})$ profiles were evaluated by re-running the simulation trajectories with Gromacs version 4.0.2 and applying the Irving-Kirkwood contour as used in other works and which is detailed in Ref. 33. Cubic splines were used to smooth the obtained profiles.

Mass density, lateral pressure and electrostatic potential profiles were computed by averaging production runs as long as $24 \mathrm{~ns}$ (excluding periods with pore formation) to achieve the correct convergence. All of the ' $z$ '-profiles presented in this paper were plotted as a function of a rescaled ' $z$ '-axis (Z') such that the average location of phosphate groups at each leaflet was placed at $Z^{\prime}= \pm 1$. By doing so, profiles corresponding to different membrane systems could be compared.

\section{Results and Discussion}

\subsection{DMSO decreases the minimum electroporation field}

MD simulations of membrane systems for different bilayer compositions and DMSO contents have been conducted, and the values of the electroporation thresholds for the different simulated systems are provided in Table 1. As reported in Ref. 9, cholesterol, by means of its condensing and ordering effect, increases the compactness of the bilayer, thus increasing the strength of the electric field required to porate it. A similar effect has been recently found in MD simulations 
when using lipid molecules with acyl chains and/or linkages (ester or ether) that favor membrane stability ${ }^{17}$ or for the addition of archaeal lipids in DPPC bilayers. ${ }^{18}$

More importantly, and as the main contribution of this paper, the addition of DMSO to the solvent phase notably decreases the minimum required electroporating field and also the corresponding voltage for a given membrane system (Table 1). This effect is significant even at very low DMSO doses, such as $1 \mathrm{~mol} \%$. It is clear, then, that the presence of DMSO favors electroporation; in other words, that the effects of the two physical and chemical poration modes are additive, and they cooperate to form water pores in the bilayer.

The analysis of the two poration mechanisms reveals that both have a similar effect on the ability of water to penetrate the membrane. Since this effect is consistent regardless of the bilayer Chol contents, in the remaining of the paper only the case with 20 mol\% of Chol has been analyzed (the results for the Chol-free and $40 \mathrm{~mol} \%$ Chol membrane display similar qualitative results). Different simulated DOPC/20\% Chol membrane systems have been examined and compared in detail to unveil the main characteristics of the poration process: a reference case corresponding to the unperturbed system ( $0 \mathrm{~mol} \% \mathrm{DMSO}, \mathrm{E}=0 \mathrm{mV} / \mathrm{nm})$, a membrane subjected to an electroporating external field ( $\left.0 \mathrm{~mol} \% \mathrm{DMSO}, \mathrm{E}=\mathrm{E}_{0}=475 \mathrm{mV} / \mathrm{nm}\right)$, a set of bilayers with specified amounts of DMSO in the solvent phase (1, 2.5 and $5 \mathrm{~mol} \%$ DMSO, E=0 mV/nm), and a mixed situation in which DMSO is present and an electroporating field is applied (5 mol\% DMSO, $E=E_{0}=150 \mathrm{mV} / \mathrm{nm}$ ). Although the analysis presented here is based on the membrane transversal properties, it is worth providing the modifications of the main structural membrane characteristics due to DMSO and/or an external electric field. In the reference case, the DOPC $/ 20 \%$ Chol total membrane area is $\mathrm{A}_{\text {tot }}=44.5 \mathrm{~nm}^{2}$, the area per DOPC molecule is $\mathrm{A}_{\mathrm{PC}}=0.63$ $\mathrm{nm}^{2}$, and the bilayer thickness measured as the average distance between phosphate groups in 
opposite leaflets is $4.17 \mathrm{~nm} .{ }^{59}$ The application of an external field does not change these properties substantially; only slight membrane expansion $\left(A_{\text {tot }}=45.1 \mathrm{~nm}^{2}, A_{P C}=0.64 \mathrm{~nm}^{2}\right)$ and thinning $(4.09 \mathrm{~nm})$ are observed. ${ }^{9}$ Instead, the addition of $5 \mathrm{~mol} \%$ DMSO significantly increases the membrane area $\left(\mathrm{A}_{\mathrm{tot}}=58.1 \mathrm{~nm}^{2}, \mathrm{~A}_{\mathrm{PC}}=0.84 \mathrm{~nm}^{2}\right)$ and reduces its thickness $(3.60 \mathrm{~nm}){ }^{5}$ For the mixed case, the effect of DMSO is dominant $\left(\mathrm{A}_{\text {tot }}=59.1 \mathrm{~nm}^{2}, \mathrm{~A}_{\mathrm{PC}}=0.85 \mathrm{~nm}^{2}\right.$ and $3.54 \mathrm{~nm}$ in thickness).

\subsection{Alteration of mass density profiles}

The initial step of pore formation is the generation of water fluctuations/defects; namely, groups of water molecules that slightly penetrate the hydrophobic region of the bilayer and can either retract, recovering the original interphase shape, or advance, forming a thin cone of water molecules that approaches the bilayer center. In the latter case, the water intrusion may progress and eventually reach the opposite leaflet, forming a hydrophobic water pore as a precursor of a stable hydrophilic pore once lipid headgroups rotate and stabilize the water column. ${ }^{6-9,13,31}$ The occurrence of water fluctuations can be generally associated to the water density in the interfacial membrane/water region and can be analyzed using the mass density profiles (see Fig. 1). As a general behavior (reference case), the lipid profile shows the typical features obtained from diffraction experiments: a pronounced lipid density peak in the phosphate group position and a minimum in the middle of the bilayer connected by a plateau in the region where cholesterol resides. Water is prevented from entering the hydrophobic membrane region, and its density profile is strongly reduced at distances below the phosphate location.

When the electric field is applied, the density profiles are not greatly altered, with the exception that insertion of water in the membrane is significantly enhanced (Fig. 1). When 
DMSO is added to the system, it accumulates in the interfacial membrane/water region, and due to its amphiphilic character and small size, it causes a lipid disordering $\operatorname{effect}^{5}$ (lipid profiles are smoothed), and it also greatly enhances water insertion (Fig. 1). The mixed case displays similar alterations of the density profiles, particularly that of water penetration. Note that in this latter case, although the applied field is lower than the applied field in the case without DMSO (150 $\mathrm{mV} / \mathrm{nm}$ vs $475 \mathrm{mV} / \mathrm{nm}$ ), water penetrates more deeply inside the membrane, reflecting the strong ability of DMSO to enhance water fluctuations at the membrane interface even at lower applied electric fields. Water insertion can also be characterized by the variation of free energy as a function of the position of a water molecule along the bilayer normal, the so-called potential of mean force (PMF). This function can be computed from the mass density profiles using $P M F(z)=-k_{B} T \ln \left(\rho_{w} / \rho_{w, 0}\right)$, where $\rho_{w, 0}$ is the average water density in the bulk phase. A large barrier to the PMF implies that the membrane is rather impermeable to water. The PMF profiles are plotted in the inset of Fig. 1 for the four analyzed membrane systems and reproduce the results reported above: both DMSO and an external applied field make the membrane more permeable to water, and the effect of the electric field is strongly enhanced when DMSO is added even if the applied field is reduced.

\subsection{Alteration of lateral pressure profiles}

Water intrusion into the hydrophobic region of a lipid bilayer is generally regulated by its compactness or cohesive nature. This property can be quantified by the local lateral membrane pressure profiles. In Fig. 2, the pressure profiles are plotted for the analyzed bilayer systems. The reference DOPC/20\% Chol case displays the typical shape of membrane pressure profiles. In the 
inner region of the membrane, the repulsion between the hydrocarbon segments leads to positive pressures that eventually result in the largest positive peak at the inner membrane/water interface where the more ordered segments close to the lipid headgroups interact the most. Another positive pressure peak is found at the phosphate location due to the repulsion among the lipid headgroups. Importantly, membrane cohesion is provided by the prominent negative pressure peak localized at the interfacial membrane/water region as a consequence of the large surface tension between water and the lipid hydrocarbon tails. The cohesion of a membrane depends on its lipid composition, as revealed by MD simulations of membranes made of different lipid species and compositions. For instance, the use of lipids with methyl groups in their hydrophobic tails and/or ether instead of ester linkages result in more stable membranes and increase the electroporation threshold. ${ }^{17}$ Addition of archaeal lipids strongly compact lipid bilayers and also increases $\mathrm{E}_{0}{ }^{18} \mathrm{~A}$ similar effect is found for cholesterol: inclusion of cholesterol in a lipid bilayer causes a condensing/ordering effect that results in greater membrane cohesion that in turn increases the minimum electroporating field $\mathrm{E}_{0}$, as it has been also supported by electromechanical rupture experiments. ${ }^{34}$ Electroporation thresholds for Chol-containing membranes are computed from MD simulations (Table 1 and Ref. 9). Comparing the two unperturbed bilayer systems (DOPC vs DOPC/20\%Chol) in Fig. 2, the cohesive effect of cholesterol is demonstrated by the increase of the negative pressure peak in the cholesterolcontaining membrane.

The application of an external electric field is found to reduce the negative pressure peak at the membrane/water interface (Fig. 2), thus explaining the greater water insertion inside the membrane. In the context of lipid bilayers subjected to electric fields, it is known that the applied field induces a compressive stress perpendicular (inward) to the bilayer surface that results in 
membrane expansion and therefore in a loss of membrane compactness ${ }^{35,36}$ that explains the observation reported above. With respect to DMSO, this effect on the reduction of membrane cohesion is extremely relevant: the addition of 1 mol\% DMSO more than compensates for the cohesive effect due to the $20 \mathrm{~mol} \%$ cholesterol in the membrane and larger amounts (2.5 and 5 mol\% DMSO) reduce the negative pressure peak even more (Fig. 2). As has been noted in the mass density profiles, DMSO molecules mostly reside at the membrane-water interface due to their amphiphilic nature and small size, thus relaxing the membrane/water line tension and facilitating the formation of water fluctuations that penetrate the lipid membrane. In the mixed case, the two effects are additive, and the negative pressure peak is extremely reduced (Fig. 2).

\subsection{Alteration of electrostatic profiles}

Another important issue that has to be analyzed when studying electroporation and chemical poration mechanisms is related to the electrostatic membrane behavior. In the absence of any perturbation, the typical membrane electrostatic potential profile is determined by the interplay between the dipoles of the system components at the membrane/water interface. Two main contributions have to be considered: the headgroup DOPC dipole, characterized by the vector that connects the phosphate (-) and choline (+) groups (the PN dipole vector), and the water dipole. The contribution of other dipoles, such as that of the hydroxyl group of cholesterol, is negligible. Electric field profiles are computed by the integral $E(z)=\int_{z=0} \frac{\rho_{q}(z)}{\varepsilon_{0}} d z$, starting at the bilayer center and moving outwards in the direction of the bilayer normal for each leaflet. The field profiles are plotted for the reference case in the upper panel of Fig. 3 for the total system, as well as the contributions due to DOPC and water molecules. These contributions reveal that at 
the membrane/water interface of both leaflets, the PN headgroup dipole vectors point outwards, whereas the water dipoles are aligned in the opposite direction, with their $\mathrm{H}$ atoms pointing towards the membrane. In the inner region of the interface, $\left|Z^{\prime}\right| \approx 0.75$, and the negative charge of phosphate groups dominates; whereas at longer distances from the bilayer center, $\left|Z^{\prime}\right| \approx 1$, and the positive charge region of the aligned water dipoles reverses this behavior. The electrostatic potential profile therefore changes its curvature, $\frac{d^{2} V}{d z^{2}}=-\frac{d E}{d z}$, from positive to negative when traversing the interface region of each bilayer leaflet in the outwards direction (see the reference case in the lower panel of Fig. 3). These observations provide the electrostatic origin for the impermeability of lipid bilayers to water according to the following argument. The force exerted in the z-direction on water molecules due to the electric field gradient is given by $F_{z}=\mu_{z} \frac{d E}{d z}$, where $\mu_{z}$ is the z-component of the water dipole moment. ${ }^{6}$ Because the water dipoles are rather aligned and point to the membrane at each leaflet at the interface, the effect of a negative electric field gradient at the inner region of the interface, $\left|Z^{\prime}\right| \approx 0.75$, is to push the water molecules outwards, preventing them from occupying deeper regions of the membrane.

The two poration mechanisms studied here alter the electrostatic membrane behavior to break the above-mentioned electrostatic 'barrier' to water penetration. The application of a transverse electric field has a direct and strong influence on the PN and water dipole alignments. A comparison between the thin and thick solid lines in the upper panel of Figure 3 reveals two different alterations. At the anodic side $\left(+, Z^{\prime}<0\right)$, the water alignment is enhanced, whereas the headgroup dipoles become more disordered than in the unperturbed situation. At the cathodic side $\left(-, Z^{\prime}>0\right)$, the headgroup dipole alignment is reinforced, whereas some water molecules are 
reversed to follow the external field. These two effects increase water penetration. At the anodic side, the negative field gradient at the inner interface region, $Z^{\prime} \approx-0.75$, disappears upon application of the external field (black solid lines in the upper panel of Fig. 3), so the aligned water molecules are pushed inside the membrane inner region. At the cathodic side, the negative field gradient region is not abolished but pushes the water molecules that reverse their orientation inwards upon application of the external field.

The addition of DMSO also breaks the electrostatic 'barrier' to water penetration. DMSO mostly resides at the inner membrane/water interface region, and because it has an electric dipole, it strongly alters the electric field and electrostatic potential profiles at this location. The green curve in the upper panel of Fig. 3 shows that the DMSO dipoles are aligned inwards; namely, they are preferentially oriented in such a way that they present their O atoms to the lipid polar groups and place their methyl groups in the more hydrophobic region of the interface. The new contribution to the total electric field and electrostatic potential profiles almost suppresses the negative field gradient region (upper panel of Fig. 3) and the positive potential curvature region (lower panel of Fig. 3) at both leaflets so that the electrostatic 'barrier' to water penetration is greatly reduced. In other words and using an equivalent argument, the presence of the hydrophilic parts of aligned DMSO molecules at the inner membrane/water interface region provides a more hydrophilic 'environment' to promote water insertion that may eventually generate membrane pores.

\subsection{DMSO stabilizes transient hydrophobic pores}

In addition to the role of DMSO in promoting water fluctuations as the initial step of electropore formation, this compound also assists the electroporation process at more advanced 
stages. The preferential location and orientation of DMSO molecules stabilizes not only the presence of water molecules in the inner interfacial membrane region but also the ensuing unstable (hydrophobic) water columns..$^{6-9}$ At this stage, DMSO wraps the water column with a sort of envelope that acts as a surfactant so that its hydrophobic character is tempered. Visual inspection of the formation of an electropore in the simulated mixed case (5 mol\% DMSO, $\mathrm{E}=\mathrm{E}_{0}=150 \mathrm{mV} / \mathrm{nm}$ ) shows the surfactant action of DMSO at this intermediate stage of electroporation (see Fig. 4). By lowering the interfacial energy of the transient water column with the surrounding hydrophobic lipid tails, it becomes stable for a longer time, allowing extra time for the lipid headgroups to rotate and form a stable hydrophilic water pore.

\section{Conclusions}

MD simulations have been used to show that the addition of DMSO facilitates electroporation in different simple cholesterol-containing lipid bilayers. According to the reported observations, chemical poration and electroporation phenomena display similar poration mechanisms. Both the application of an external electric field and the addition of DMSO greatly alter the two typical transversal properties of a lipid bilayer in a way that promotes water fluctuations at the membrane/water interface and favors pore initiation. Therefore, when acting together, DMSO and external electric fields enable membrane pore formation to be achieved under more favorable conditions. The molecular characteristics of DMSO provide this compound with particular abilities to assist the electroporation process. Three DMSO effects have been suggested to explain the favorable role of this compound in electroporation. First, due to their amphiphilic character and small size, DMSO molecules tend to be located at the membrane- 
water interface, thus relaxing the negative lateral pressure peak that compacts the bilayer structure and facilitating the intrusion of water fluctuations into the lipid membrane. Second, due to its dipole moment, DMSO strongly alters the electrostatic membrane profile in a way that promotes the progress of the initial stages of electroporation. Finally, DMSO molecules act as a surfactant and relax the surface tension when the intermediate hydrophobic water pore is formed.

Experimental evidence of the increase of electroporation efficiency by DMSO has already been described by Klempt et al., ${ }^{19}$ but no further systematic research or development has been attempted since then. The results reported here suggest plausible improvements in the electroporation techniques by the use of DMSO and open the possibility of reducing the strength of the applied electroporating fields and/or of electroporating cells that are difficult to porate. We hope that our findings encourage experimentalists to investigate the advantages of using DMSO (or other small, amphiphilic and polar compounds) in the electroporation of both model in vitro lipid bilayers and living cell membranes.

ACKNOWLEDGMENTS Computational resources were provided by the Barcelona Supercomputing Center and by the Centro de Cómputos de Alto Rendimiento (CeCAR) Facultad de Ciencias Exactas y Naturales - UBA. Financial support was provided by SEID through project BFU2010-21847-C02-02, by DURSI through project 2009-SGR-1055 and by grants from Universidad de Buenos Aires (UBACyT 2011), CONICET (PIP 2009, PIP 2012), and MINCyT (SLO-AR 08/02/09). MLF gratefully acknowledges the guidance of Professor G. Marshall. 


\section{REFERENCES}

(1) Tsong, T. Y. Electroporation of cell membranes. Biophys. J. 1991, 60, 297-306.

(2) Neumann, E.; Kakorin, S.; Toensing, K. Fundamentals of electroporative delivery of drugs and genes. Bioelectrochem. Bioenerg. 1999, 48, 3-16.

(3) M. Belehradek, M.; Domenge, C.; Luboinski, B.; Orlowski, S.; Belehradek, J.; Mir, LL. M. Electrochemotherapy, a new antitumor treatment. Cancer 1993, 72, 3694-3700.

(4) Kligman, A. M. Topical pharmacology and toxicology of dimethylsulfoxide. J. Am. Med. Assoc. 1965, 193, 194-195.

(5) de Ménorval, M. -A.; Mir, LL. M.; Fernández, M. L.; Reigada, R. Effects of Dimethyl Sulfoxide in Cholesterol-Containing Lipid Membranes: A Comparative Study of Experiments In Silico and with Cell. PLoS One 2012, 7, e41733.

(6) Tieleman, D. P. The molecular basis of electroporation. BMC Biochem. 2004, 5, 10.

(7) Tarek, M. Membrane electroporation: a molecular dynamics study. Biophys. J. 2005, 88, 4045-4053.

(8) Levine, Z. A.; Vernier, P. T. Life cycle of an electropore: field-dependent and fieldindependent steps in pore creation and annihilation. J. Membrane Biol. 2010, 236, 27-36.

(9) Fernández, M. L.; Marshall, G.; Sagués, F.; Reigada, R. Structural and kinetic molecular dynamics study of electroporation in cholesterol-containing bilayers. J. Phys. Chem. B 2010, $114,6855-6865$. 
(10) Gurtovenko, A. A.; Anwar, J.; Vattulainen, I. Defect-Mediated Trafficking across Cell Membranes: Insights from In Silico Modeling. Chem. Rev. 2010, 110, 6077-6103.

(11) Gurtovenko, A. A.; Anwar, J. Modulating the structure and properties of cell membranes: the molecular mechanism of action of dimethyl sulfoxide. J. Phys. Chem. B 2007, 111, 1045310460.

(12) Notman, R.; Noro, M. G.; O’Malley, B.; Anwar, J. Molecular basis for dimethylsulfoxide (DMSO) action on lipid membranes. J. Am. Chem. Soc. 2006, 128, 13982-13983.

(13) Tokman, M.; Lee, J. H.; Levine, Z. A.; Ho, M.-C.; Colvin, M. E.; Vernier, P. T. Electric Field-Driven Water Dipoles: Nanoscale Architecture of Electroporation. PLoS One 2013, 8, e61111.

(14) Ho, M.-C.; Casciola, M.; Levine, Z. A.; Vernier, P. T. Molecular Dynamics Simulations of Ion Conductance in Field-Stabilized Nanoscale Lipid Electropores. J. Phys. Chem. B 2013, $117,11633-11640$.

(15) Casciola, M.; Bonhenry, D.; Liberti, M.; Apollonio, F.; Tarek, M. A molecular study of cholesterol rich lipid membranes: comparison of electroporation protocols. Bioelectrochemistry 2014, in press.

(16) Dehez, F.; Delemotte, L.; Kramar, P.; Miklavčič, D.; Tarek, M. Evidence of Conducting Hydrophobic Nanopores Across Membranes in Response to an Electric Field. J. Phys. Chem. C 2014, 118, 6752-6757. 
(17) Polak, A.; Bonhenry, D.; Dehez, F.; Kramar, P.; Miklavčič, D.; Tarek, M. On the Electroporation Thresholds of Lipid Bilayers: Molecular Dynamics Simulation Investigations. $J$. Membrane Biol. 2013, 246, 843-850.

(18) Polak, A.; Tarek, M.; Tomšič, M.; Valant, J.; Ulrih, N. P.; Jamnik, A.; Kramar, P.; Miklavčič, D. Electroporation of archaeal lipid membranes using MD simulations. Bioelectrochemistry 2014, (in press).

(19) Melkonyan, H.; Sorg, C.; Klempt, M. Electroporation Efficiency in Mammalian Cells is increased by Dimethyl Sulfoxide (DMSO). Nucleic Acids Research 1996, 24, 4356-4357.

(20) Lindahl, E.; B. Hess, B.; van der Spoel, D. GROMACS 3.0: a package for molecular simulation and trajectory analysis. J. Mol. Model. 2001, 7, 306-317.

(21) Berger, O.; Edholm, O.; Jahnig, F. Molecular dynamics simulations of a fluid bilayer of dipalmitoylphosphatidylcholine at full hydration, constant pressure, and constant temperature. Biophys. J. 1997, 72, 2002-2013.

(22) Martinez-Seara, H.; Róg, T.; Karttunen, M.; Reigada, R.; Vattulainen, I. Influence of cis double-bond parameterization on lipid membrane properties: how seemingly insignificant details in force-field change even qualitative trends. J. Chem. Phys. 2008, 129, 105103.

(23) Berendsen, H. J. C.; Postma, J. P. M.; van Gunsteren, W. F.; Hermans, J. Interaction models for water in relation to protein hydration, in Intermolecular Forces. B. Pullman: Reidel, Dordrecht, The Netherlands, 1981. 
(24) Holtje, M.; Forster, T.; Brandt, B.; Engels, T.; von Rybinski, W.; Holtje, H. -D. Molecular dynamics simulations of stratum corneum lipid models: fatty acids and cholesterol. Biochim. Biophys. Acta 2001, 1511, 156-167.

(25) Miyamoto, S.; Kollman, P. A. SETTLE: an analytical version of the SHAKE and RATTLE algorithms for rigid water models. J. Comput. Chem. 1992, 13, 952-962.

(26) Hess, B.; Bekker, H.; Berendsen, H. J. C.; Fraaije, J. G. E. M. LINCS: a linear constraint solver for molecular simulations. J. Comput. Chem. 1997, 18, 1463-1472.

(27) Essman, U.; Perera, L.; Berkowitz, M. L.; Darden, H. L. T.; Pedersen, L. G. A smooth particle mesh Ewald method. J. Chem. Phys. 1995, 103, 8577-8592.

(28) Berendsen, H. J. C.; Postma, J. P. M.; van Gunsteren, W. F.; DiNola, A.; Haak, J. R. Molecular dynamics with coupling to an external bath. J. Chem. Phys. 1984, 81, 3684-3690.

(29) Martinez-Seara, H.; Róg, T.;. Pasenkiewicz-Gierula, M.; Vattulainen, I.; Karttunen, M.; Reigada, R. Interplay of unsaturated phospholipids and cholesterol in membranes: effect of double-bond position. Biophys. J. 2008, 95, 3295-3305.

(30) Bordat, P.; Sacristan, J.; Reith, D.; Girard, S.; Glätti, A. et al. An improved dimethyl sulfoxide force field for molecular dynamics simulations. Chem. Phys. Lett. 2003, 374, 201-205.

(31) Delemotte, L.; Tarek, M. Molecular Dynamics Simulations of Lipid Membrane Electroporation. J. Membrane Biol. 2012, 245, 531-543. 
(32) Gurtovenko, A. A.; Vattulainen, I. Calculation of the electrostatic potential on lipid bilayers from molecular dynamics simulations: Methodological issues. J. Chem. Phys. 2009, $130,215107$.

(33) Ollila, S.; Hyvönen, M.T.; Vattulainen, I. Polyunsaturation in lipid membranes: dynamic properties and lateral pressure profiles. J. Phys. Chem. B 2007, 111, 3139-3150.

(34) Koronkiewicz, S.; Kalinowski, S. Influence of cholesterol on electroporation of bilayer lipid membranes: chronopotentiometric studies. Biochim. et Biophys. Acta 2004, 1661, 196-203.

(35) Crowley, J. M. Electrical breakdown of bimolecular lipid membranes as an electromechanical instability. Biophys. J. 1973, 13, 711-724.

(36) Needham, D.; Hochmuth, R. M. Electro-mechanical permeabilization of lipid vesicles. Role of membrane tension and compressibility. Biophys. J. 1989, 55, 1001-1009. 
Table 1. Minimum electroporating field $E_{0}$ and the generated voltage $V_{0}$ for the three simulated bilayer systems upon the addition of different amounts of DMSO to the solvent phase.

\begin{tabular}{|c|c|c|c|}
\hline \multirow{3}{*}{0 mol\% Chol } & mol\% DMSO & $\begin{array}{c}E_{0} \\
(\mathrm{mV} / \mathrm{nm})\end{array}$ & $\begin{array}{c}V_{0} \\
(\mathrm{~V})\end{array}$ \\
\hline \multirow{3}{*}{0} & 0 & 325 & 2.60 \\
\cline { 2 - 4 } & 1 & 200 & 1.52 \\
\cline { 2 - 4 } & 2.5 & 150 & 1.07 \\
\hline \multirow{3}{*}{20} & 5 & 75 & 0.49 \\
\cline { 2 - 4 } & 1 & 475 & 3.95 \\
\cline { 2 - 4 } & 2.5 & 225 & 2.63 \\
\cline { 2 - 4 } & 5 & 150 & 1.08 \\
\hline \multirow{3}{*}{40} & 0 & 750 & 6.47 \\
\cline { 2 - 4 } & 1 & 600 & 5.02 \\
\cline { 2 - 4 } & 2.5 & 450 & 3.75 \\
\cline { 2 - 4 } & 5 & 350 & 2.87 \\
\hline
\end{tabular}

\section{FIGURE CAPTIONS}

Figure 1. Mass density profiles for DOPC/20\%Chol bilayer systems averaged and plotted as a function of the absolute value of the scaled distance Z'. The profiles correspond to DOPC (black), Chol (green), water (blue), DMSO (violet) and DOPC phosphate groups (red). Inset: membrane permeability profiles averaged and plotted as a function of IZ'I for the cases shown in the main figure. In both figures, thin solid lines correspond to $0 \mathrm{~mol} \% \mathrm{DMSO}$ and $\mathrm{E}=0 \mathrm{mV} / \mathrm{nm}$, thin dashed lines correspond to $5 \mathrm{~mol} \% \mathrm{DMSO}$ and $\mathrm{E}=0 \mathrm{mV} / \mathrm{nm}$, thick solid lines correspond to 
$0 \mathrm{~mol} \% \mathrm{DMSO}$ and $\mathrm{E}=475 \mathrm{mV} / \mathrm{nm}$, and thick dashed lines correspond to $5 \mathrm{~mol} \% \mathrm{DMSO}$ and $\mathrm{E}=150 \mathrm{mV} / \mathrm{nm}$.

Figure 2. Lateral pressure profiles $\mathrm{P}_{\mathrm{L}}\left(\left|Z^{\prime}\right|\right)$ plotted as a function of the re-scaled bilayer normal axis $\mid Z$ 'l for different simulated membrane systems. The results in solid lines correspond to DOPC/20\%Chol membranes under the influence of DMSO and/or an external electric field (see legends). The profile for the pure DOPC unperturbed membrane is plotted with a dashed line.

Figure 3. Electric field $E\left(Z^{\prime}\right)$ (upper panel) and electrostatic potential V(Z') (lower panel) profiles plotted as a function of the re-scaled bilayer normal axis $Z^{\prime}$ for DOPC/20\%Chol membranes under the influence of DMSO and/or an external electric field.

Figure 4. Snapshot in the $(y, z)$ view of the initial formation of a hydrophobic pore in a DOPC/ $20 \%$ Chol membrane under the effects of $5 \mathrm{~mol} \%$ DMSO in the solvent phase and an external electric field of $\mathrm{E}=150 \mathrm{mV} / \mathrm{nm}$ applied in the normal bilayer plane direction. The hydrophobic pore develops into a stable hydrophilic pore once the lipid headgroups rotate, but before this happens, DMSO molecules 'protect' the water column. Water and DMSO molecules are plotted with cyan and yellow sticks, respectively. For clarity, lipid molecules are not plotted. Only the phosphorous atom groups of DOPC and the hydroxyl groups are visualized using red and orange beads, respectively. 


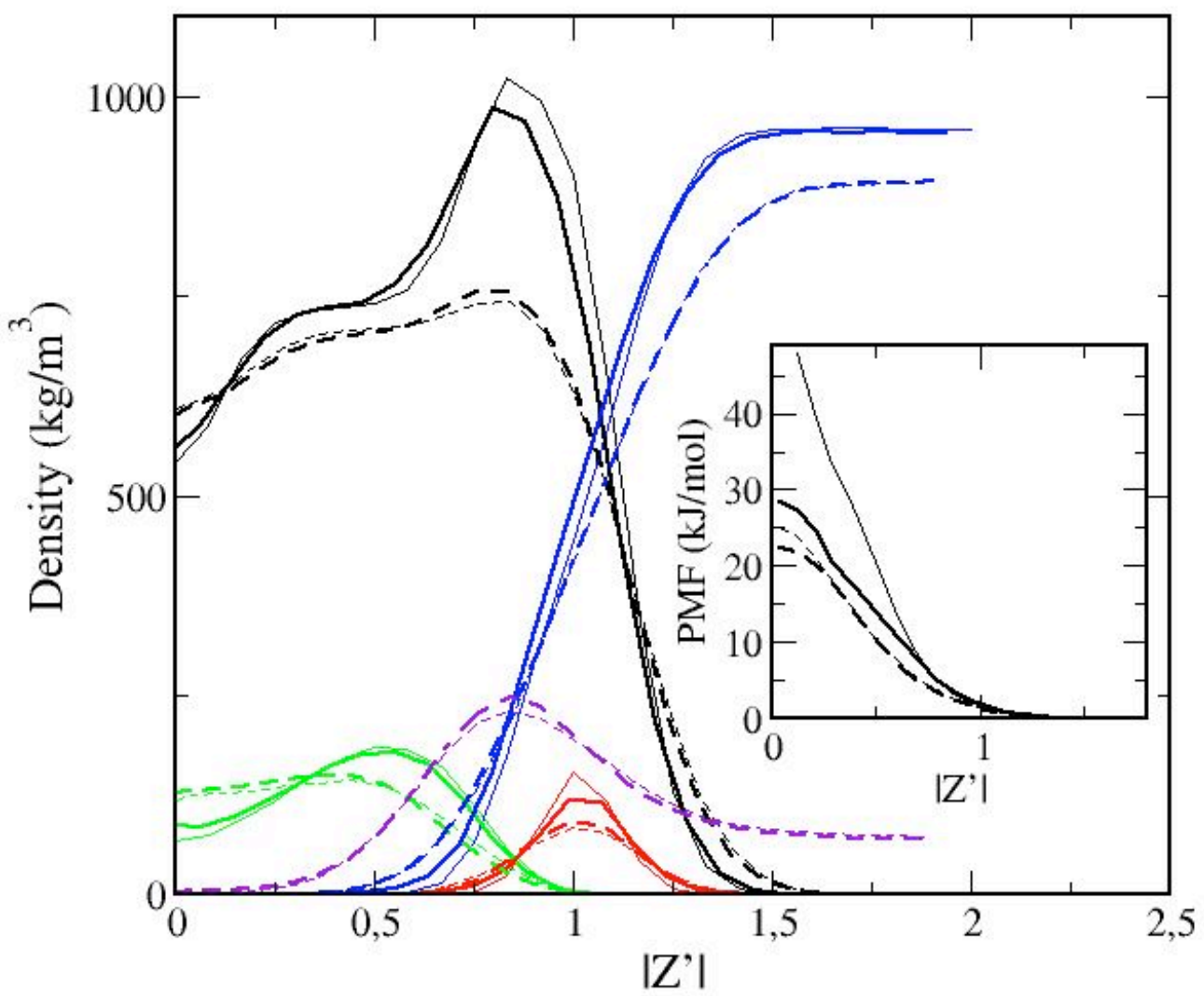

Figure 1. 


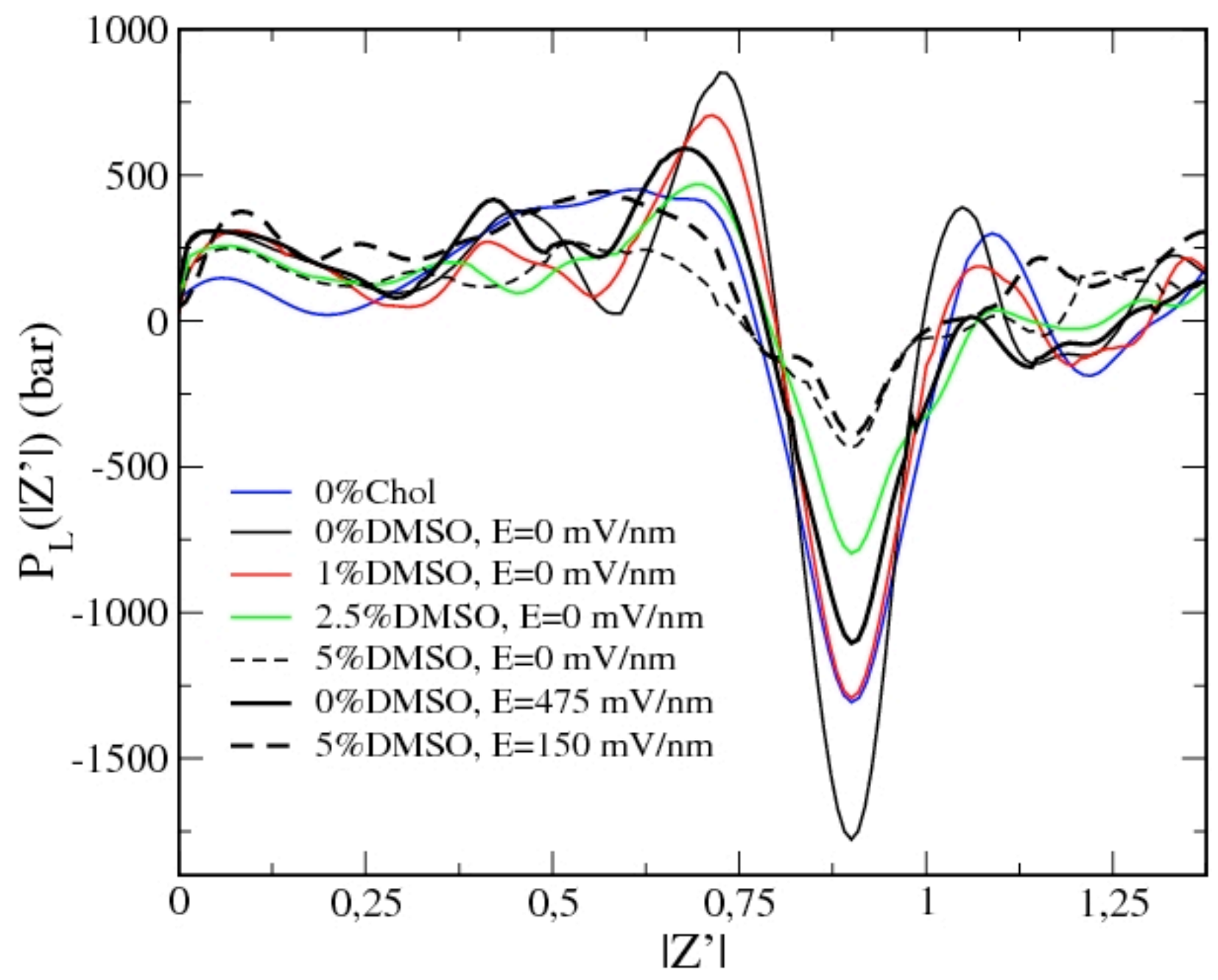

Figure 2. 


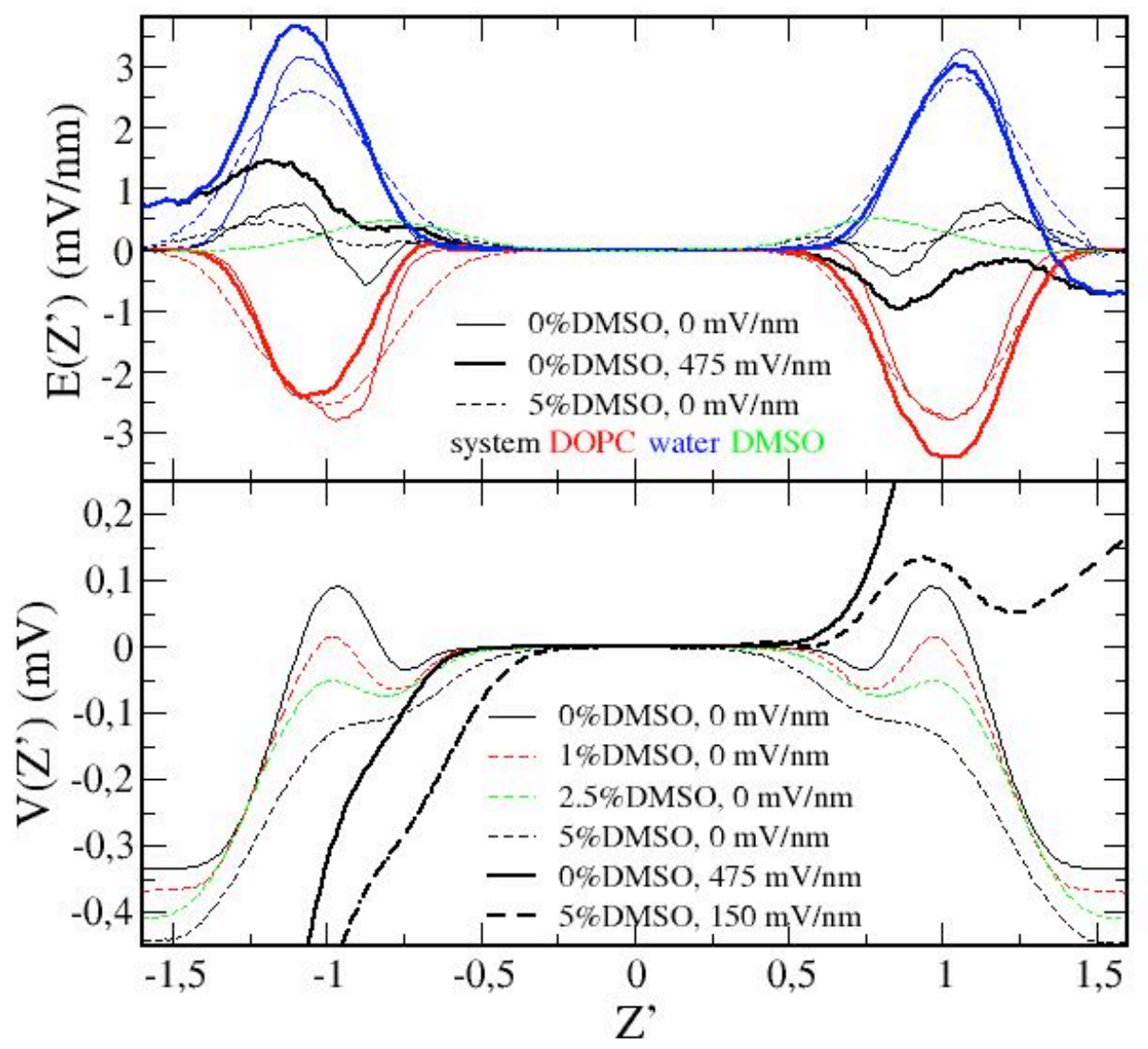

Figure 3. 


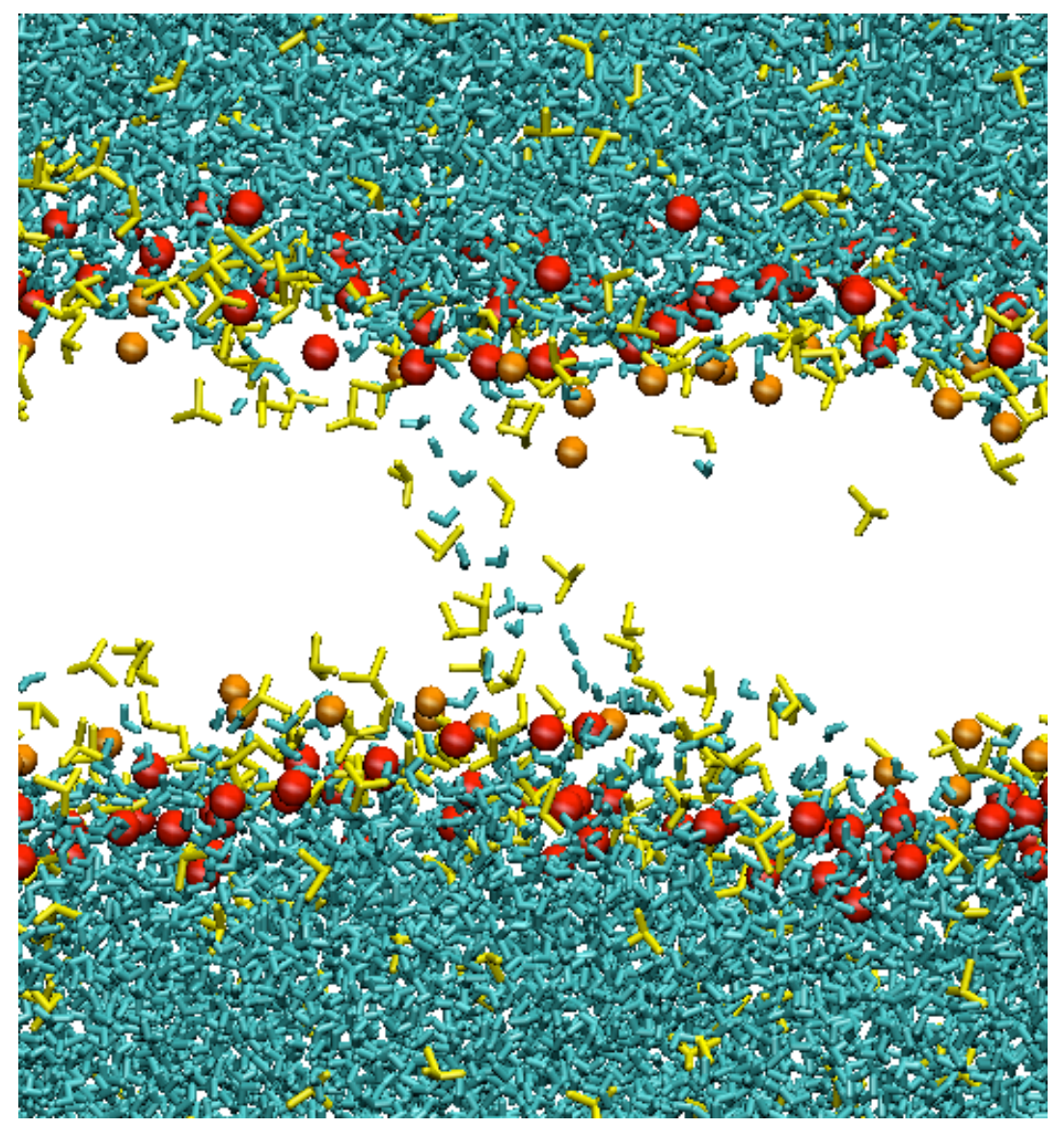

Figure 4. 


\section{Table of Contents (TOC) Image}
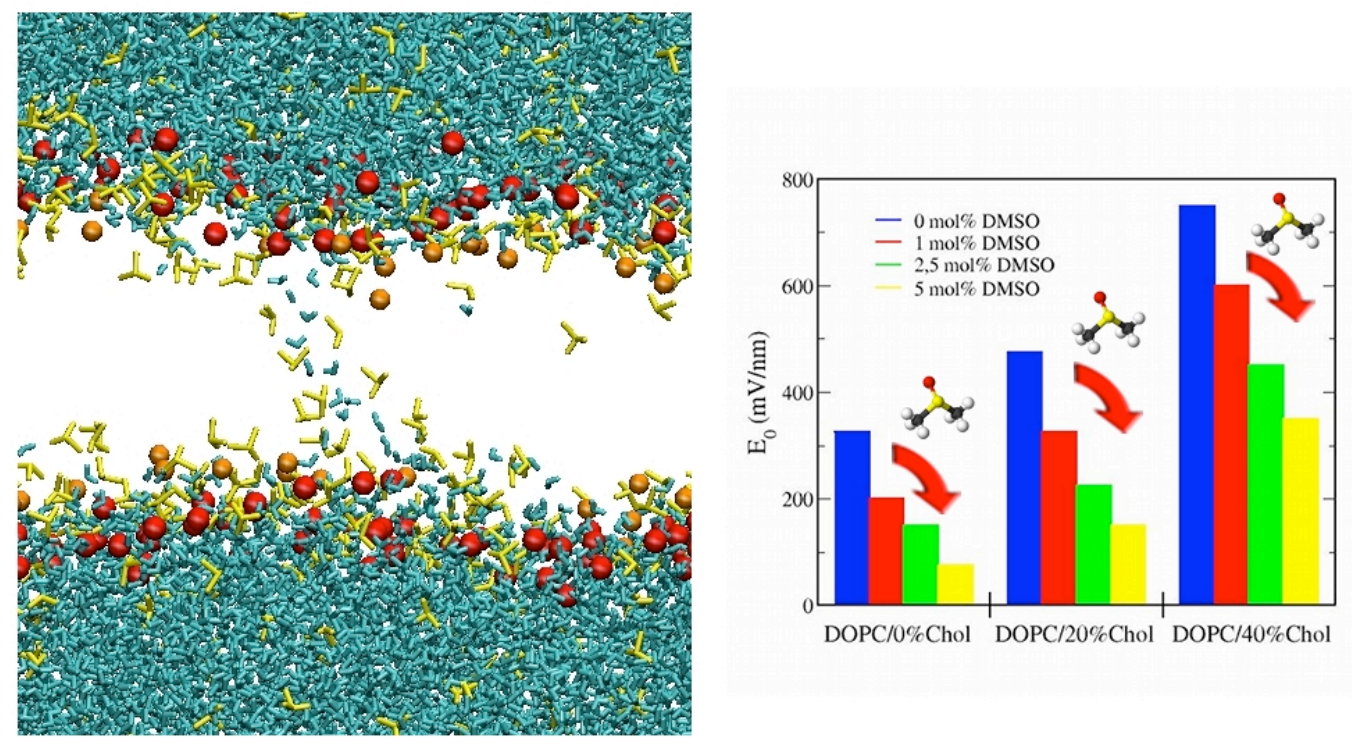